\title{
Figure-of-merit for phase-change materials used in thermal management
}

\author{
Lei Shao, ${ }^{1}$ Arun Raghavan, ${ }^{2,3}$ Gun-Ho Kim, ${ }^{1,4}$ Laurel Emurian, ${ }^{2}$ Jeffrey Rosen, ${ }^{5}$ \\ Marios C. Papaefthymiou, ${ }^{5}$ Thomas F. Wenisch, ${ }^{5}$ Milo M. K. Martin, ${ }^{2}$ and Kevin P. Pipe ${ }^{1,5^{*}}$ \\ ${ }^{1}$ Mechanical Engineering, University of Michigan, Ann Arbor, MI 48109-2125 \\ ${ }^{2}$ Computer and Information Science, University of Pennsylvania, Philadelphia, PA 19104-6309 \\ ${ }^{3}$ Oracle Labs, Belmont, California 94002 \\ ${ }^{4}$ Materials Science and Engineering, University of Michigan, Ann Arbor, MI 48109-2125 \\ ${ }^{5}$ Electrical Engineering and Computer Science, University of Michigan, Ann Arbor, MI 48109-
}

*Corresponding author: pipe@umich.edu.

\begin{abstract}
Phase-change materials (PCMs) may be utilized to thermally manage short bursts of high-power heat fluxes. In this work, we numerically investigated the figure-of-merit $(F O M)$ for PCMs in such applications which have complicated boundary conditions that do not have analytical solutions. Because this FOM only depends on material thermophysical properties, it provides a metric to compare the performance of various PCMs in managing high-power heat flux over a short timescale. We also experimentally demonstrate, for the first time, the use of a PCM with relatively high $F O M$ on a timescale as short as 1 second for a heat flux as high as $1.1 \times 10^{5} \mathrm{~W} / \mathrm{m}^{2}$ in real-world electronic devices.
\end{abstract}

\section{Keywords}


Phase-change materials (PCMs); Figure-of-merit (FOM); Metallic alloys; Phase-change heat sinks; Computational sprinting; Thermal management; Heat sinks.

\section{Nomenclature}

$C_{P} \quad$ specific heat capacity $(\mathrm{J} /(\mathrm{kg} \cdot \mathrm{K}))$

FOM figure-of-merit $\left(\mathrm{W}^{2} \cdot \mathrm{s} /\left(\mathrm{K} \cdot \mathrm{m}^{4}\right)\right)$

$h \quad$ latent heat $(\mathrm{J} / \mathrm{kg})$

$h_{C} \quad$ convection heat transfer coefficient $\left(\mathrm{W} /\left(\mathrm{K} \cdot \mathrm{m}^{2}\right)\right)$

$q$ heat flux $\left(\mathrm{W} / \mathrm{m}^{2}\right)$

$q_{0}$ heat flux constant $\left(\mathrm{W} \cdot \mathrm{s}^{1 / 2} / \mathrm{m}^{2}\right)$

$S t_{L} \quad$ Stefan number, liquid (dimensionless)

$t \quad$ melting or cooldown time (s)

$T_{0} \quad$ initial temperature $\left({ }^{\circ} \mathrm{C}\right)$

$T_{f} \quad$ melting temperature $\left({ }^{\circ} \mathrm{C}\right)$

TDP thermal design power $(\mathrm{W})$

$u_{0} \quad$ initial temperature relative to the melting point $\left({ }^{\circ} \mathrm{C}\right)$

$u_{\mathrm{L}} \quad$ boundary temperature relative to the melting point $\left({ }^{\circ} \mathrm{C}\right)$

$x \quad$ distance within the phase change materials (m)

Greek letters

$\alpha_{\mathrm{L}} \quad$ liquid thermal diffusivity $\left(\mathrm{m}^{2} / \mathrm{s}\right)$ 
$\kappa_{\mathrm{L}} \quad$ liquid thermal conductivity $(\mathrm{W} /(\mathrm{m} \cdot \mathrm{K}))$

$\lambda \quad$ dimensionless parameter

$\rho \quad \operatorname{density}\left(\mathrm{kg} / \mathrm{m}^{3}\right)$

\section{Introduction}

Phase-change materials (PCMs) have been extensively studied for numerous applications in thermal management and storage by absorbing or releasing latent heat when they changes from solid to liquid and vice versa. Conventional PCMs, such as paraffins [1] and salt hydrates [2], have been widely used on the timescales of several hours in systems as small as lithium-ion batteries [3, 4], and as large as chilled food/medicine transport [5], solar energy storage systems $[6,7]$, and buildings [8]. However, recent studies have proposed using PCMs to target short bursts of high-power heat flux in the sub-second timescale, prompting studies of PCMs with a much smaller thermal time constant [9-11]. Among them, one example which has attracted a great deal of attention recently suggests to use PCMs for storing excessive heat generated during intense computation from mobile devices (e.g., cellular phones, tablets), which briefly ( 1 second) exceeds the maximum steady-state power dissipation by a magnitude order, known as computational sprinting [11-14], aimed to improve responsiveness for bursty computational demands in devices restricted by passive cooling.

Using conventional PCMs on this timescale is challenging, since their low thermal conductivities dominate the thermal process. Numerous works have been done to improve the thermal conductivity of PCMs, such as examining the use of metal or diamond microchannels or mesh carriers [15, 16], and suspended graphite or graphene enhanced hybrid materials [17-19], 
etc. Furthermore, there are also emerging interests in metallic alloys due to their high thermal conductivity and reliable melting points [20]. While there are many different types of pristine and engineered PCMs, very few studies have investigated the figure-of-merit $(F O M)$ to quantitatively compare various PCMs for their performance in dealing with high-power heat flux in a short timescale. Until most recently, P.J. Shamberger analytically developed a FOM by generalizing the two-phase Stefan problem of melting of a semi-infinite material with a fixed boundary temperature [21]. While the accurate FOM depends on the initial and boundary temperatures, it could be approximated to only contain material thermophysical properties under the situations in which sensible heat (absorbing heat by changing temperature) is negligible in comparison to latent heat (absorbing heat by changing phase). This approximation is valid because sensible heat is usually minimized in phase-change cooling systems to avoid temperature surge.

In this work, we focus on the study of FOMs for PCMs used in conditions of high-power heat flux by numerical and experimental approaches. While the FOM is developed through analytical derivation by P.J. Shamberger, explicit solutions only exist for a few ideal boundary conditions. As a result, we used numerical approaches here to investigate several realistic conditions such as constant heat fluxes, non-constant boundary temperatures, finite-size PCM layers, and intermittent heat-flux boundary conditions. This is representative of how PCMs are used in real-world thermal management applications, and has not been studied so far. We also numerically investigated sensible heat which is neglected in the derivation of the approximate FOM so that we provide a real analysis of the problem as a whole.

For the first time, we integrate PCMs in a real mobile electronic device to buffer the heat generated by its microprocessors. We also perform the first experimental demonstration using 
PCMs on the timescale as short as 1 second by using a PCM with relatively high FOM. Further, we investigate PCMs subject to non-constant boundary conditions like short-timescale thermal spikes numerically and experimentally. By studying such an intermittent thermal process, we reveal new design insights for thermal management of power electronics which are typically operated by a discontinuous pulsed fashion, e.g., computational sprinting.

Section 2 validates the approximate $F O M$ by numerical simulations for conditions that analytical solutions do not exist, including non-constant boundary temperatures, geometries with finite size and intermittent heat fluxes. Section 3 describes the experimental setup and procedure of studying the thermal consequences of integrating PCMs with different FOMs into systems subject to high-power thermal spikes (computational sprinting operation). Section 4 presents and discusses experimental results. Finally, we conclude this work in Section 5.

\section{Phase-change figure-of-merit}

Phase-change problems were first generalized by Stefan in 1889 [22] followed by numerous analytical skills [23-25] developed for solving special types of boundary conditions without significant insights into the material performance itself. Until recently, P.J. Shamberger studied the Stefan problem from the perspective towards the performance of PCMs and analytically developed a figure-of-merit (FOM) for the cooling capacity of PCMs [21]. Because it is a function of the thermophysical properties of a PCM, it has important implications for design and optimization of PCMs for thermal systems with high-power heat fluxes. Here, for a clear description of the problem studied in this work, we start by restating the solutions of a onedimensional Stefan problem for a semi-infinite PCM occupying $x>0$ at uniform initial temperature $T_{0}$ which is no higher than the melting point $T_{f}$. To simplify the comparison among 
PCMs with different $T_{f}$, we use temperature difference relative to $T_{f}$, so that the initial temperature can be expressed as $u_{0}=T_{0}-T_{f}$. Explicit analytic solutions only exist if the heat source is at a constant temperature (constant boundary temperature $u_{\mathrm{L}}$ ), for which the heat flux at the boundary $x=0$ satisfies $q=q_{0} \cdot \mathrm{t}^{-1 / 2}$. Convection in the melted region is not considered here. $u_{\mathrm{L}}$ has been derived as [21]:

$$
u_{L}=\frac{q_{0}}{\kappa_{L}} \sqrt{\pi \cdot \alpha_{L}} \operatorname{erf}(\lambda),
$$

where $\kappa_{\mathrm{L}}$ is the liquid thermal conductivity, $\alpha_{\mathrm{L}}$ is the liquid thermal diffusivity $\left(\alpha_{\mathrm{L}}=\kappa_{\mathrm{L}} /\left(\rho \cdot C_{P}\right)\right.$ in which $\rho$ is the density and $C_{P}$ is the specific heat capacity), erf is the error function, and $\lambda$ is a dimensionless parameter given by the following transcendental equation if $u_{0}$ is negligibly small:

$$
\frac{\sqrt{\pi}}{S t_{L}} \cdot \lambda \cdot \operatorname{erf}(\lambda)=e^{-\lambda^{2}}
$$

where $S t_{L}=\frac{C_{P} u_{L}}{h}$ is the liquid Stefan number in which $h$ is the specific latent heat. The assumption that $u_{0}$ is negligibly small means that sensible heat is negligible in comparison to latent heat. Because PCMs are used for applications where the latent heat is not overwhelmed by the heat flux which also implies that the liquid Stefan number is small, the term $\frac{\sqrt{\pi}}{S t_{L}}$ in Eq. (2) is usually much greater than 1 and $\lambda$ is much smaller than 1 . Therefore, $\operatorname{erf}(\lambda) \approx \lambda \approx \sqrt{\frac{S t_{L}}{\sqrt{\pi}}}<1$.

Substituting into Eq. (1) yields

$$
u_{L}=\frac{q_{0}^{2}}{\kappa_{L} \cdot \rho \cdot h} \sqrt{\pi}
$$


To minimize the boundary temperature $u_{\mathrm{L}}$, the product $\kappa_{\mathrm{L}} \cdot \rho \cdot h$ should be maximized. Since this product only involves material thermophysical properties, it is the $F O M$ which can be used to quantitatively compare the performance of different types of PCMs. This is the same metric that has been derived by P.J. Shamberger [21] as the approximate FOM while considering over a small temperature range near their melting point. Table 1 shows the FOMs for several different types of popular PCMs; their thermophysical data are obtained from reference [2].

\begin{tabular}{|c|c|c|c|c|c|}
\hline Material type & $\begin{array}{c}\text { Melting } \\
\text { Temperature } \\
T_{f}\left({ }^{\circ} \mathrm{C}\right)\end{array}$ & $\begin{array}{l}\text { Latent Heat } \\
h\left(\times 10^{3} \mathrm{~J} / \mathrm{kg}\right)\end{array}$ & $\begin{array}{c}\text { Thermal } \\
\text { Conductivity } \\
\kappa_{\mathrm{L}}(\mathrm{W} /(\mathrm{m} \cdot \mathrm{K}))\end{array}$ & $\begin{array}{l}\text { Density } \\
\rho\left(\mathrm{kg} / \mathrm{m}^{3}\right)\end{array}$ & $\begin{array}{c}F O M \\
\left(\times 10^{3} \mathrm{~W}^{2} \cdot \mathrm{s} /\right. \\
\left.\left(\mathrm{K} \cdot \mathrm{m}^{4}\right)\right)\end{array}$ \\
\hline $\begin{array}{l}\text { Inorganics } \\
\qquad \mathrm{MgCl}_{2} \cdot 6 \mathrm{H}_{2} \mathrm{O}\end{array}$ & 117 & 168.6 & 0.570 & 1450 & 139 \\
\hline $\begin{array}{c}\mathrm{Mg}\left(\mathrm{NO}_{3}\right)_{2} \cdot \\
6 \mathrm{H}_{2} \mathrm{O}\end{array}$ & 89 & 162.8 & 0.490 & 1550 & 123 \\
\hline $\mathrm{Ba}(\mathrm{OH})_{2} \cdot 8 \mathrm{H}_{2} \mathrm{O}$ & 48 & 265.7 & 0.653 & 1937 & 336 \\
\hline $\mathrm{CaCl}_{2} \cdot 6 \mathrm{H}_{2} \mathrm{O}$ & 29 & 190.8 & 0.540 & 1562 & 160 \\
\hline $\begin{array}{l}\text { Organics } \\
\quad \text { Paraffin wax }\end{array}$ & 64 & 173.6 & 0.167 & 790 & 22 \\
\hline Polyglycol E600 & 22 & 127.2 & 0.189 & 1126 & 27 \\
\hline $\begin{array}{l}\text { Fatty acids } \\
\quad \text { Palmitic acid }\end{array}$ & 64 & 185.4 & 0.162 & 850 & 25 \\
\hline Capric acid & 32 & 152.7 & 0.153 & 878 & 20 \\
\hline Caprylic acid & 16 & 148.5 & 0.149 & 901 & 19 \\
\hline $\begin{array}{c}\text { Metallic alloys } \\
\text { Roto136F }\end{array}$ & 58 & 28.9 & 10 & 9010 & 2603 \\
\hline
\end{tabular}


Here we first compare the FOMs of two PCMs which will be used for experimental investigation later. One is paraffin wax (Blended Waxes, BW-407), a conventional PCM, with $T_{f}$ in the range from $54{ }^{\circ} \mathrm{C}$ to $58{ }^{\circ} \mathrm{C}$ (assumed a sharp $T_{f}$ at $58{ }^{\circ} \mathrm{C}$ for simplicity). Wax is typically known [1] to have $h=200 \times 10^{3} \mathrm{~J} / \mathrm{kg}, \rho=800 \mathrm{~kg} / \mathrm{m}^{3}, C_{P}=2200 \mathrm{~J} /(\mathrm{kg} \cdot \mathrm{K})$, and $\kappa_{\mathrm{L}}=0.2 \mathrm{~W} /(\mathrm{m} \cdot \mathrm{K})$. The other one is an alloy (Rotometals, Roto136F) composed of $49 \% \mathrm{Bi}, 21 \% \mathrm{In}, 18 \% \mathrm{~Pb}$ and $12 \%$ Sn (mass percentages) with a $T_{f}$ of $58{ }^{\circ} \mathrm{C}$. This alloy is known [26] to have $h=28.9 \times 10^{3} \mathrm{~J} / \mathrm{kg}, \rho$ $=9010 \mathrm{Kg} / \mathrm{m}^{3}, C_{P}=185 \mathrm{~J} /(\mathrm{kg} \cdot \mathrm{K})$, and $\kappa_{\mathrm{L}}=10 \mathrm{~W} /(\mathrm{m} \cdot \mathrm{K})$. We note that although there are several lead-free alloys available near this melting temperature, Roto136F is used in this work because of its well-known material parameters. Under the condition of a high-power heat flux $q=1 \times 10^{5}$. $\mathrm{t}^{-1 / 2} \mathrm{~W} / \mathrm{m}^{2}$, for the alloy, because $\lambda$ is much smaller than $1(\lambda=0.15)$ due to its high $F O M$, Eq. (3) is valid to estimate $u_{\mathrm{L}}$. However, for the paraffin wax which is overwhelmed by the high heat flux because of its low FOM, $\lambda$ is big enough $(\lambda=0.87)$ such that $\operatorname{erf}(\lambda)$ in Eq. (1) is close to 1 . Substituting $\operatorname{erf}(\lambda) \approx 1$ into Eq. (1) yields

$$
u_{L}=\frac{q_{0} \sqrt{\pi}}{\sqrt{\kappa_{L} \cdot \rho \cdot C_{P}}} .
$$

Eq. (4) converts to the well-known condition that the Stefan number is high and latent heat is small. It shows that latent heat is not involved to cool a heat source with a heat flux overwhelming the FOM of the selected PCM. Instead, exploiting sensible heat of materials with high thermal conductivity and specific heat is more efficient. This also suggests that it is not effective by integrating PCMs right on a high-power-density heat source, such as at the junction of power electronics, where the heat flux could usually reach $10^{9} \mathrm{~W} / \mathrm{m}^{2}$ [9]. Rather, PCMs (even 
with relatively high FOMs) should be kept at the other side of the chip, which can spread the heat from the microscale or nanoscale junction to the whole chip area before it reaches the PCMs.

While the above $F O M$ is developed through analytical derivation, explicit solutions only exist for a few ideal boundary conditions. Therefore, numerical approaches are used below to investigate several realistic conditions such as non-constant boundary temperatures, finite-size PCM layers, and intermittent heat-flux boundary conditions. We first study a constant heat-flux boundary condition (resulting in non-constant boundary temperatures) by numerically calculating a semi-infinite PCM with two different constant heat fluxes $q=1 \times 10^{4} \mathrm{~W} / \mathrm{m}^{2}$ and $q=$ $1 \times 10^{5} \mathrm{~W} / \mathrm{m}^{2}$ applied at $x=0$ and with the initial condition of a uniformly distributed temperature $u_{0}=0$ at solid phase. Fig. 1 (a) shows $u_{\mathrm{L}}$ (after 1 second) as a function of both FOMs $\left(26 \times 10^{6}-\right.$ $\left.26000 \times 10^{6} \mathrm{~W}^{2} \cdot \mathrm{s} \cdot \mathrm{K}^{-1} \cdot \mathrm{m}^{-4}\right)$ and $C_{P}(40-4000 \mathrm{~J} /(\mathrm{Kg} \cdot \mathrm{K}))$, which demonstrates that varying $C_{P}$ does not affect $u_{\mathrm{L}}$ at all if the applied constant heat flux is $q=1 \times 10^{4} \mathrm{~W} / \mathrm{m}^{2}$. In Fig. 1 (b), after we increase the heat flux by an order of magnitude, we show that $C_{P}$ is still not related in the region of high FOMs, which result in a small Stefan number required by the aforementioned approximation in deriving Eq. 3. Therefore, the approximate FOM holds for most situations where the PCMs are not overwhelmed by the heat flux. Two PCMs are marked in Fig. 1 (b); one is the paraffin wax (BW-407) and the other is the metallic alloy (Roto136F). 
(a)

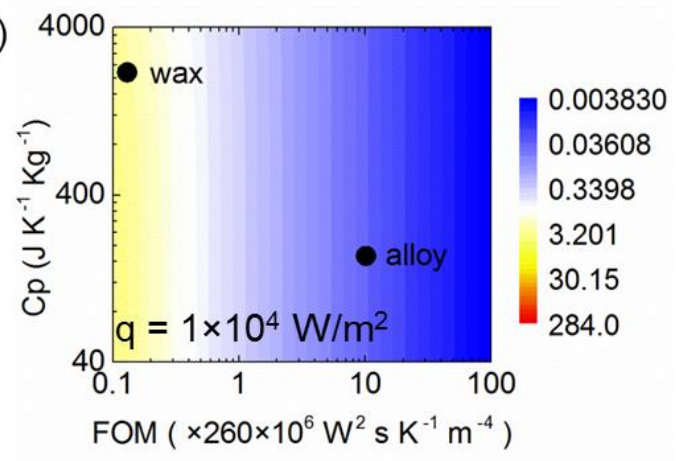

(b)

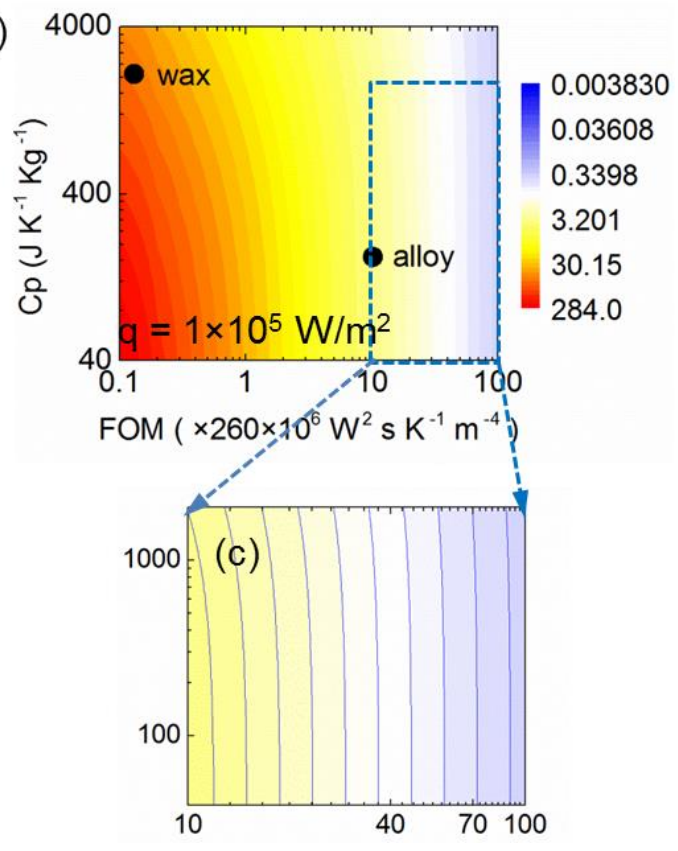

Figure 1. Temperature $u_{\mathrm{L}}$ at the boundary $x=0$ of a semi-infinite PCM heated by a constant heat flux $q$ applied at $x=0$ after a certain burst length $t$ (1 second), as a function of $F O M$ and $C_{P}$. The constant heat flux is $1 \times 10^{4} \mathrm{~W} / \mathrm{m}^{2}$ in (a) and $1 \times 10^{5} \mathrm{~W} / \mathrm{m}^{2}$ in (b). The zoom-in for the region of high FOMs of (b) is shown in (c) in which $u_{\mathrm{L}}$ is demonstrated as independent on $C_{P}$. 

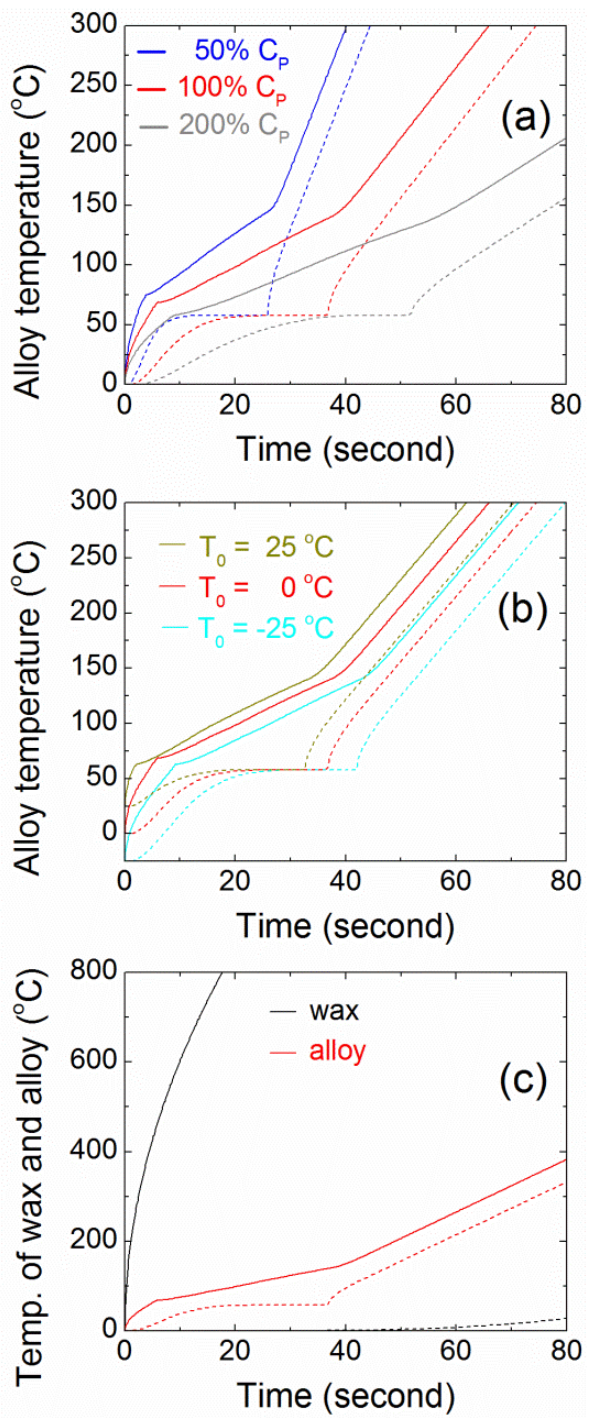

Figure 2. Temperatures at $x=0$ (solid line) and at $x=1 \mathrm{~cm}$ (dashed line) of the 1D layer of PCM. (a) Sensible heat is varied by sweeping the $C_{P}(50 \%, 100 \%$, and $200 \%)$ of the alloy Roto136F while the initial temperatures are set the same. (b) Sensible heat is varied by sweeping the initial temperature $\left(-25^{\circ} \mathrm{C}, 0{ }^{\circ} \mathrm{C}\right.$, and $\left.25^{\circ} \mathrm{C}\right)$ of the alloy Roto136F while the $C_{P}$ are set the same. (c) Comparison of the temperatures between wax (high $C_{P}$ but low $F O M$ ) and alloy (low $C_{P}$ but high FOM) for the same initial and boundary conditions. 
In the above analytical derivation of the $F O M$, the sensible heat is practically neglected so that it is not treated. This is, first of all, because the sensible heat could be designed to be easily minimized in real-world phase-change thermal management applications. Second, phasechange equations are highly nonlinear as the sensible heat is involved in a transcendental equation which itself depends on material properties. The sensible heat is also related with the initial and final temperatures which are determined by the boundary conditions, not solely by the material thermophysical properties. As a result, including sensible heat in the FOM makes it not immediately clear how much and how sensitive it depends on material properties. However, in order to analyze the problem as a whole and to investigate the impact of sensible heat on phasechange thermal management applications, we numerically calculated a 1D PCM layer in which the amount of sensible heat involved in pre-melt and post-melt processes is varied. The PCM is a thermally insulated 1D layer with thickness of $1 \mathrm{~cm}$ and initial condition of uniformly distributed temperature below the melting point $\left(58^{\circ} \mathrm{C}\right)$. At one side of the 1D layer $(x=0)$, a constant heat flux $10^{5} \mathrm{~W} / \mathrm{m}^{2}$ was turned on at time $t=0$. Fig. 2 compares the temperatures of the $1 \mathrm{D}$ layer.

Several insights may be gained based on Fig. 2, as discussed below. First, pre-melt sensible heat is the penalty paid by phase-change thermal management applications to utilize latent heat. For PCMs that have high FOMs and are therefore able to effectively cool a heat source (PCMs with low FOMs do not melt quickly and sufficiently), the amount of sensible heat (pre-melt penalty) determines the time at which the phase transition begins; a larger sensible-heat penalty delays the phase transition (Figs. 2 (a) and (b)). Second, for PCMs that have high FOMs (such as the alloy Roto136F), for a given heat source, the average PCM temperature at the end of the phase transition is almost the same even for different amounts of sensible heat (Figs. 2 (a) and (b)). Third, after the phase transition is completed, the temperature obviously rises much 
faster for a PCM with smaller specific heat capacity (Fig. 2 (a)). But this rise could be avoided by carefully choosing a material and/or mass with sufficient latent heat for known (given) thermal patterns. Fourth, a larger specific heat capacity is always preferred as it stores more sensible heat under similar temperature change (grey curve in Fig. 2 (a)). However, empirically, most PCMs with a large specific heat capacity, such as paraffin wax and water/ice, usually suffer from a low phase-change FOM (Fig. 2 (c)) and are therefore not appropriate as a buffer for highpower thermal spikes regardless of their high specific heat capacity. Finally, phase-change thermal management systems can be co-designed with transient heat sources of known frequency and duty cycle such that the high heat flux duration consumes nearly all of the PCM's latent heat and the PCM re-solidifies between heat pulses, to minimize the sensible heat penalty. To summarize the above points, sensible heat should be considered when designing phase-change thermal management systems (for which design variables may include the heating pattern, initial and final temperatures, etc.), while the analytically derived FOM is important when selecting a particular PCM.

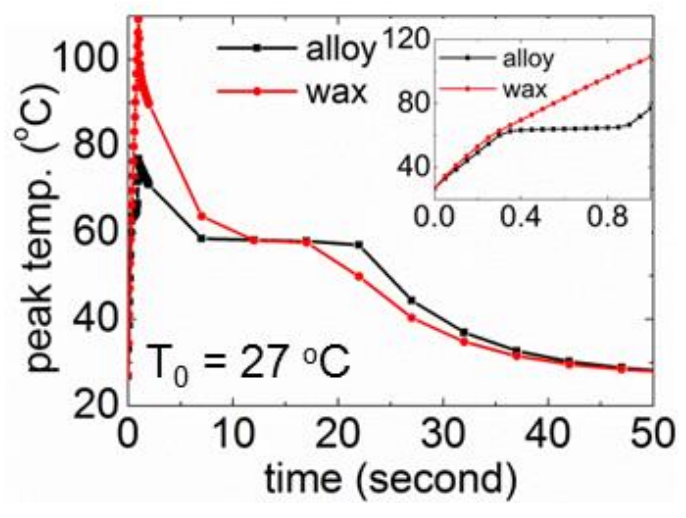

Figure 3. Temperature of the silicon layer shows complete cycles of an alloy/silicon stack and a wax/silicon stack (same geometry) heated by a constant heat flux turned on at $t=0$ and turned off at $t=1 \mathrm{~s}$; cooling is provided by natural convection with air. 
More important, in order to investigate the complete cycle (including heating and cooldown) of PCMs with different FOMs dealing with intermittent heat fluxes (e.g., high-power thermal spikes), we then study a thin layer of PCM by performing a finite element analysis using the Comsol Multiphysics package. The layered structure studied here is consisting of $0.325 \mathrm{~mm}$ thick silicon and $0.2 \mathrm{~mm}$ thick PCM (consistent with the experiments shown later). We added convection $h_{C}=10 \mathrm{~W} /\left(\mathrm{K} \cdot \mathrm{m}^{2}\right)$ at both sides of the PCM/silicon structure and a constant heat flux $q=1 \times 10^{5} \mathrm{~W} / \mathrm{m}^{2}$ at the surface of the silicon. The initial temperature is $27^{\circ} \mathrm{C}$ and the heat flux $q$ $=1 \times 10^{5} \mathrm{~W} / \mathrm{m}^{2}$ is turned on at $t=0$ and turned off at $t=1 \mathrm{~s}$. As shown in Fig. 3, temperatures of the silicon layer are simulated for a complete heating and cooldown cycle with the alloy (Roto136F) and wax (BW-407) used as PCMs respectively. During the cooldown process, although the temperature is clamped longer at $58{ }^{\circ} \mathrm{C}$ in the case the alloy is used because of its higher volumetric latent heat, both are cooled to the initial temperature after about the same time. It clearly shows that different FOMs will not affect the duty cycle although the peak temperatures can be significantly reduced by a PCM with high FOM. This is because PCMs bring little changes to a system's heat dissipation capability, which is only determined by the cooling conditions, such as natural or forced convection with different fluids. Thus, the maximum steady-state duty cycle is only determined by the ratio of the steady-state dissipation power to the burst power. This is verified by experiments shown in the following sections.

\section{Experimental procedure}


To experimentally demonstrate that PCMs with high FOMs can be used for cooling highpower thermal spikes on the timescale as short as 1 second in practical applications, we used the alloy Roto136F for storing the excessive heat generated by the operation of computational sprinting in a real smartphone. To implement sprinting, a chip in a mobile or hand-held device would ideally offer peak power exceeding its sustainable thermal design power (TDP, which is also the maximum steady-state dissipation power) by an order of magnitude or more $[11,14]$. Because it is not realistic for existing mobile chips to work at such high power due to current chip designs and the power delivery constraints of mobile batteries, we designed a silicon thermal test chip (TTC) as a proxy for the smartphone processor with integrated PCM microreservoirs as on-chip phase-change heat sinks.

Figs. 4 (a) - (d) shows the fabrication process of these TTCs, which were made from 0.5mm-thick $\mathrm{Si}$ wafers with 0.002 -mm-thick thermally grown $\mathrm{SiO}_{2}$. On the top side of the wafer, 10/100 nm Ti/Pt thin film heaters were patterned by photolithography, metallization and lift-off, forming the test system for generating heat bursts as a proxy for a sprint-enabled smartphone chip as well as measuring the integrated heat sink's thermal response. On the bottom side, 0.2mm-deep $8 \times 8 \mathrm{~mm}^{2}$ wells were patterned by back side aligned lithography and formed by deep reactive ion etching. The wafers were then diced into $10 \mathrm{~mm} \times 10 \mathrm{~mm}$ chips, filled by Roto136F which were cut into small pieces $(0.115 \mathrm{~g})$ to completely occupy a well when melted, and encapsulate by 0.1-mm-thick copper tape. Paraffin wax BW-407 was used as a conventional PCM for comparison in separate chips. Finally, wires were soldered to the Ti/Pt heaters to operate the TTCs. Fig. 4 (e) shows a TTC without etched well, which serves as a proxy for the traditional chip design in order to provide a comparison with the integrated PCM design. 
(a)

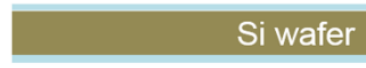

(b)

(c)
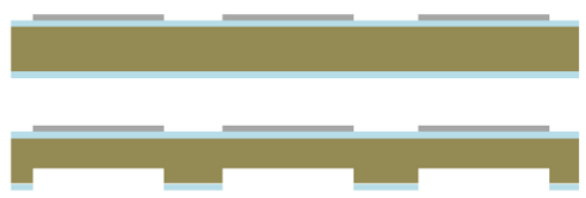

TTC with PCM

(d)

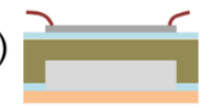

Silicon
TTC without etching

(e)

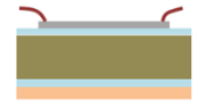

PCM Copper (f)

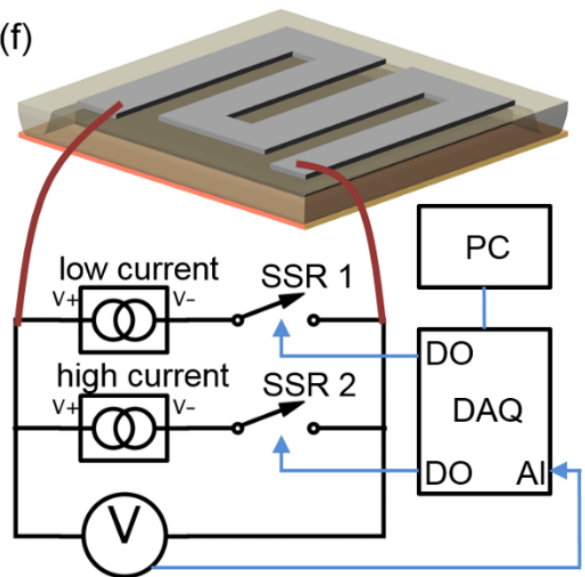

Figure 4. (a) - (d) Fabrication process of TTCs with integrated on-chip phase-change heat sinks.

(e) TTCs without etched wells or PCM filling are used for comparison. (f) Illustration of the TTC with encapsulated PCM, connected to a PC-controlled circuit used to operate the TTC.

The TTCs were operated by a DC power supply, two solid-state relays (SSRs), and a computer-controlled data acquisition (DAQ) device, as shown in Fig. 4 (f). When SSR 1 was turned on and SSR 2 was turned off, a 0.02 A constant current was applied to the Pt heater $(\sim 26$ $\Omega$ at room temperature), representing a mobile processor's idle state which is $\sim 0.01 \mathrm{~W}$. Conversely, when SSR 1 was off and the SSR 2 was on, a 0.65 A constant current was applied to deliver $\sim 11 \mathrm{~W}$. Because the resistance of the Pt thin film changes linearly with temperature and can be calibrated precisely, the measured voltage can be used to calculate the TTC temperature. In current smartphones, processor power consumption is approximately $0.01-0.02 \mathrm{~W}$ when idle and $1.2-1.3 \mathrm{~W}$ at maximum [27]. This maximum power for all smartphones is limited by the ability of steady-state natural convection to keep the surface at a temperature no greater than that of human hands $\left(\sim 35^{\circ} \mathrm{C}\right)$ [28]. Thus, the power applied to the TTC at idle was realistic, while 
the power applied during sprinting was nearly an order of magnitude greater than the current smartphone maximum, approximating ten times as many active cores under parallel computation.

\section{Results and discussions}

\subsection{TTCs suspended in air}

To study the performance of the TTC during computational sprinting, a TTC was first operated while being suspended in steady air. The low current used was $20 \mathrm{~mA}$ and the high current was $650 \mathrm{~mA}$, while the resistance of the Pt thin film was approximately $26 \Omega$ at room temperature. The idle state therefore consumed approximately $10 \mathrm{~mW}\left(1.0 \times 10^{2} \mathrm{~W} / \mathrm{m}^{2}\right)$, while the sprinting state consumed around $11 \mathrm{~W}\left(1.1 \times 10^{5} \mathrm{~W} / \mathrm{m}^{2}\right)$. Fig. 5 compares the temperature transients of three TTCs during the sprint, two filled with PCMs (BW-407 paraffin wax and Roto136F alloy, respectively) as shown in Fig. 4 (d) and the third without well-etching as shown in Fig. 4 (e). The chips were operated in sprinting mode for 0.6 second, after which they were switched to idle mode to cool down. In Fig. 5, the temperature of the chip filled with wax is much higher than the other two at the end of the sprint, and the transient shows no shoulder around the melting temperature of the wax due to its low FOM. This confirms Eq. 4 that exploring the latent heat of a PCM with low FOM (paraffin wax in this case) to target a highpower heat flux $\left(1.1 \times 10^{5} \mathrm{~W} / \mathrm{m}^{2}\right.$ in this case) is even worse than using the sensible heat (silicon in this case). 


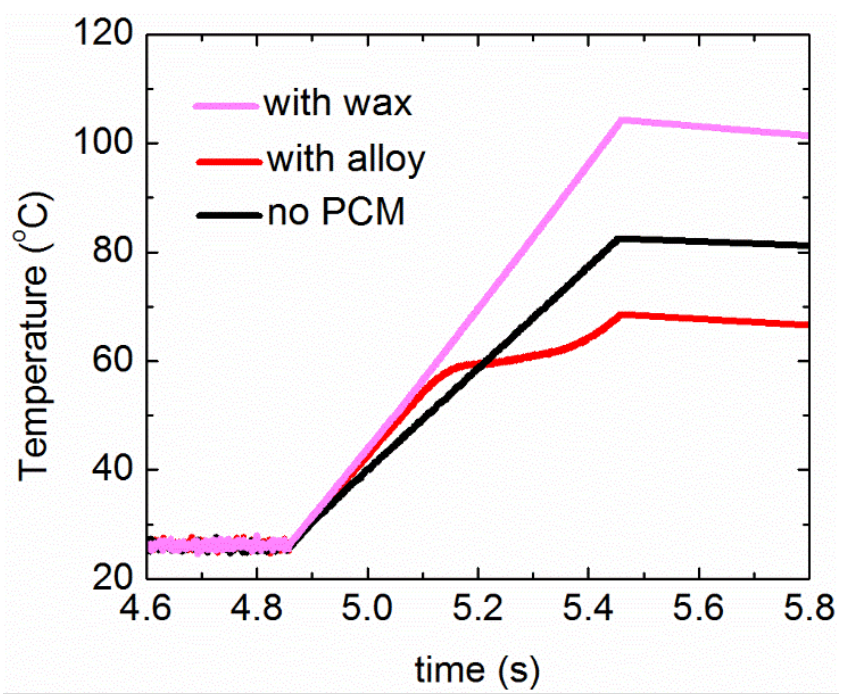

Figure 5. Comparison of the temperature transients of three TTCs (suspended in air) under computational sprinting operation, one filled with wax, one filled with alloy, and the third without integrated PCM. The applied power was turned on to sprint-mode $(11 \mathrm{~W})$ for 0.6 second and then switched to idle $(10 \mathrm{~mW})$.

We then mainly investigate the temperature transients of the TTC filled with alloy and the TTC without well-etching. In Fig. 5, the temperature of the chip filled with alloy is higher than the one without well-etching until it reaches the alloy's melting point due to both the lower specific heat capacity and lower thermal conductivity of the alloy as compared to silicon. However, for the chip filled with metallic alloy, the temperature transient has a shoulder of about 0.28 seconds at $58{ }^{\circ} \mathrm{C}$, demonstrating the metallic alloy's phase change. Based on its thermal properties, the $0.115 \mathrm{~g}$ of PCM used (measured by a precision scale) is predicted to absorb $3.32 \mathrm{~J}$ of heat during melting, translating into a 0.28 second phase change for a $\sim 11 \mathrm{~W}$ heating power, which agrees well with the experimental results. At the end of sprinting, the temperature of the TTC filled with alloy is $14^{\circ} \mathrm{C}$ lower than that of the chip with no PCM filled. The benefit of the 
PCM is reduced if the sprint is longer than the PCM melting time. We note that this integrated PCM configuration is far from optimized, and there is still significant room for improvement. The peak temperature for the TTC filled with PCM can be further decreased, for example, by increasing the amount of PCM (e.g., by etching a deeper well) or by using a different metallic alloy with a higher FOM. The paraffin wax is not used in the following experiments due to its ineffectiveness caused by its low FOM.
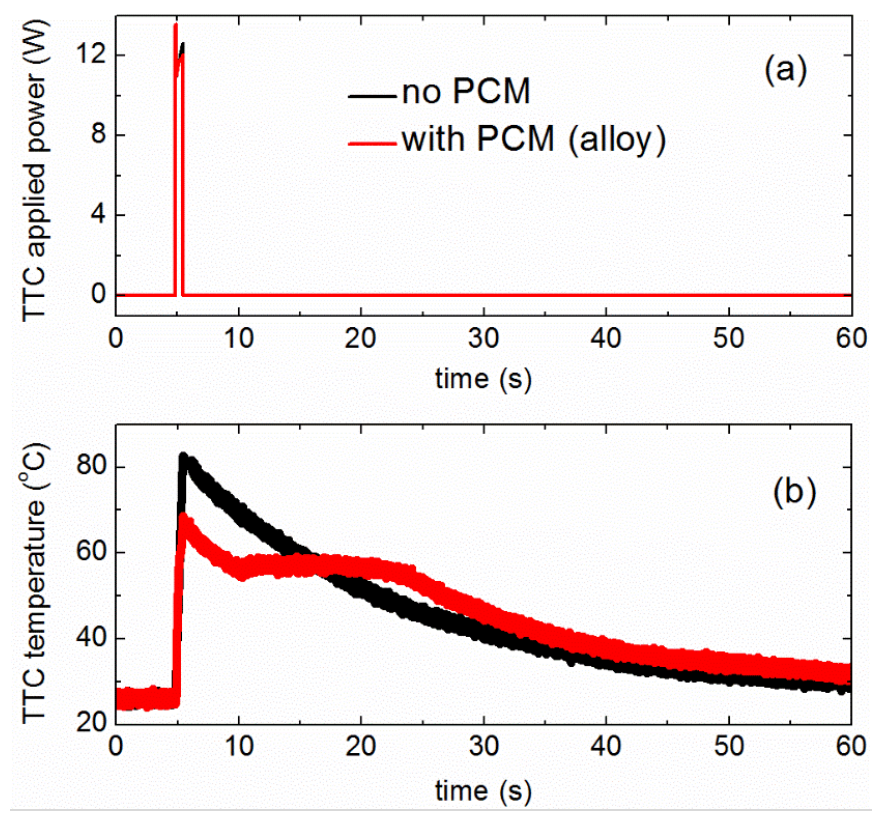

Figure 6. Comparison of the temperature transients of two TTCs (suspended in air) under sprint and cooldown operation, one filled with alloy and the other without integrated PCM.

In addition to the maximum temperature, another important metric is the cooldown time required for the chip to cool down to the initial temperature after a sprint. As shown in Fig. 6, a comparison of the cooling transients of the two chips (one filled with alloy and the other without well-etching) reveals that they return to the initial temperature after a similar time of around 1 minute, which confirms the finite element analysis in Fig. 3. This 1-minute long cooldown time 
can be significantly shortened to several seconds through the use of heat spreading materials in a smartphone, as described below.

\subsection{TDP of the TTC-mounted smartphone}

To study the performance of sprinting in an actual smartphone, a Samsung Galaxy S3 was disassembled. Between the processor package and the inner support metallic frame, there was originally a $0.6-\mathrm{mm}$-thick pad of thermal interface material, which was removed to accommodate the TTC. The TTCs described above were mounted (in separate measurements) on the inner support frame with the copper tape side (PCM side) in contact with the frame, as illustrated in Fig. 7. The two soldered lead wires extended out of the smartphone to operate the TTC. The inner support frame acts as an efficient heat spreader to dissipate the heat stored in the PCM. The smartphone was then re-assembled, suspended in steady air, powered on, and left at idle with the screen turned on in order to provide realistic background heating and thermal boundary conditions.

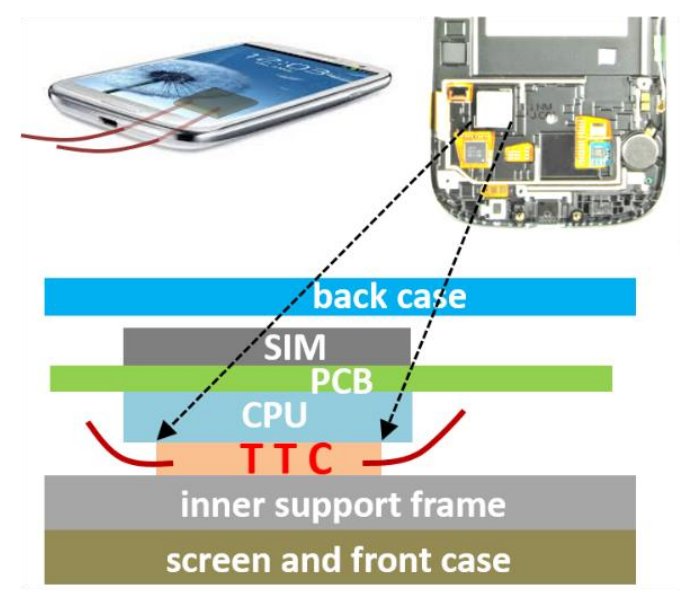

Figure 7. Smartphone with TTC mounted inside with cross-sectional view of the TTC-mounted smartphone. 
To provide a baseline for the smartphone modified to have a TTC mounted inside it, we first measured the TDP of the modified smartphone. Both the TTC filled with PCM and the other without integrated PCM were used for comparison. A thin thermocouple $(0.127 \mathrm{~mm}$ diameter $)$ was mounted on the screen's surface by silver paste at a location that was exactly above the TTC. This thermocouple measured the maximum screen temperature of the smartphone, which needs to be maintained within a comfortable range $\left(<35^{\circ} \mathrm{C}\right)$ for human hands. The temperatures of the TTC and the thermocouple were logged, while a number of different constant currents were applied to the Pt heater on the TTC for 30 minutes (separate measurements) to test the TDP until we found the proper one, which was $0.2 \mathrm{~A}(\sim 1.0 \mathrm{~W})$.

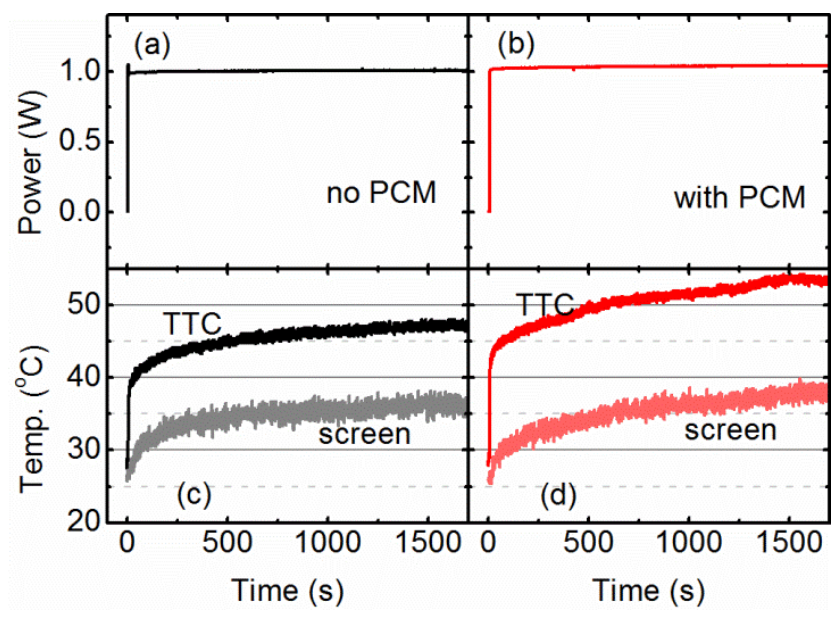

Figure 8. TDP of TTC-mounted smartphones measured by applying a constant current to the TTCs while monitoring the temperatures of both the TTC and the screen. (a) and (c) TTC shown in Fig. 4 (e) (without integrated PCM). (b) and (d) TTC shown in Fig. 4 (d) (with integrated PCM which is the alloy Roto136F studied here). 
Fig. 8 compares the temperatures for both smartphone-mounted TTCs (separate measurements) at $1.0 \mathrm{~W}$ applied power. The temperatures asymptotically reached steady state while the screen was kept no higher than the body temperature. This applied power of $1.0 \mathrm{~W}$ is the $T D P$ of the modified smartphone and also the maximum power for sustained operation, which is used for later duty cycle calculations. Here we note that the alloy did not melt because the final temperature is below its melting point; this measurement is only to obtain the TDP, not intended to melt the PCM. Fig. 8 also shows that the chip temperature is $5.5{ }^{\circ} \mathrm{C}$ higher when using the TTC with integrated PCM because the alloy sandwiched between the TTC's silicon layer and the smartphone's inner support frame has a lower thermal conductivity than silicon $(\sim$ $150 \mathrm{~W} /(\mathrm{m} \cdot \mathrm{K}))$, inducing a higher thermal resistance between the heat source and case.

\subsection{Sprint and cooldown cycling of a TTC-mounted smartphone}

Next, we operate the TTC which was mounted inside the smartphone for a complete sprint and cooldown process; one without PCM shown in black and the other with PCM shown in red. As shown in Fig. 9 (a), the peak temperature of the TTC without PCM reached $80{ }^{\circ} \mathrm{C}$ at the end of the 0.6 -second sprint while it was only $64{ }^{\circ} \mathrm{C}$ for the PCM-filled TTC. Thus, PCMs could obviously improve a chip's lifetime and reliability by keeping it at lower peak and average temperatures for the same sprinting duration $[29,30]$.

Finally we study multiple sprint and cooldown cycles by comparing TTCs with and without PCM filling and study the performance improvement induced by the integrated PCM. First we consider the case of a TTC without integrated PCM. A duty cycle $D$ (sprint length per cycle) no greater than the ratio of dissipation power to sprint power will provide an off percentage large enough so that the TTC has enough time to cool after a sprint. The TDP, 
maximum steady-state dissipation power, is measured to be $1.0 \mathrm{~W}$ in Section 4.2. Thus, given an $11 \mathrm{~W}$ sprint power, the maximum duty cycle is 1/11. Black curves in Fig. 9 (b) show the temperature transients of repeated sprints over 10 minutes with a duty cycle $D=1: 11$ (with a 0.6second sprint length and 6-second cooling time) and sprint power of $11 \mathrm{~W}$. The peak temperature of the TTC, at the end of each sprint, gradually increases from $75.6{ }^{\circ} \mathrm{C}$ to $85^{\circ} \mathrm{C}$. The red curves show the same sprint and cooldown operation while the TTC mounted in the smartphone has the on-chip integrated PCM. Near the end of the 10 minutes, it clearly shows that PCM not only reduces the peak temperature from $85{ }^{\circ} \mathrm{C}$ to $69^{\circ} \mathrm{C}$, but also reduces the rapid temperature variation from a difference of $48.6{ }^{\circ} \mathrm{C}$ to $27.3{ }^{\circ} \mathrm{C}$ (improved by $44 \%$ ) that may improve reliability associated with thermal cycling [31,32]. Thus, the performance of multiple sprint and cooldown cycles can be efficiently improved by using the on-chip integrated PCMs. 

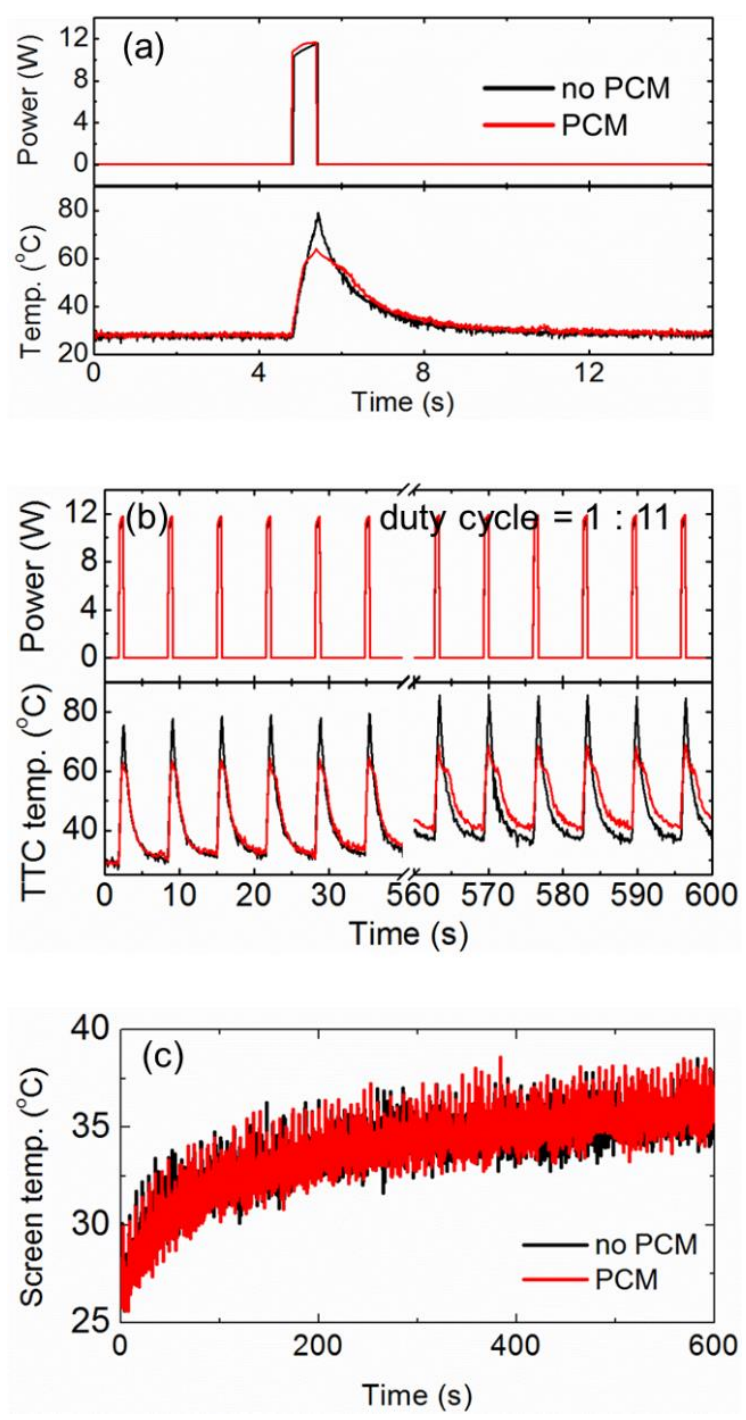

Figure 9. (a) Single sprint of smartphone-mounted TTCs, (b) power and TTC temperatures, and (c) screen temperatures for sprint and cooldown cycles with a sprinting-to-cooling ratio of 1:11 lasting for 10 minutes (only showing the first 40 seconds and the last 40 seconds in part b).

\section{Conclusion}

In this work, we first numerically investigated the FOM for PCMs proposed by prior analytical works under the condition of several realistic boundary conditions which do not have 
analytical solutions. These boundary conditions include non-constant boundary temperatures, finite size, and intermittent heat fluxes. We selected two PCMs to compare their performance dealing high-power fluxes, one conventional PCM with low FOM (a paraffin wax) and the other emerging PCM with high FOM (a metallic alloy).

We also provide the first experimental demonstration of using a PCM with high FOM in the timescale of 1 second under a heat flux as high as $1.1 \times 10^{5} \mathrm{~W} / \mathrm{m}^{2}$ using proof-of-concept PCM-filled TTCs as processor proxies in a real smartphone package. Multiple cycles of heating and cooldown were repeated to demonstrate that the low melting temperature metallic alloy PCMs can provide a thermal buffer during intermittent heating. Directly integrating the PCM with the chip mounted inside a smartphone enables the melting and cooldown process to completely occur within only a few seconds. By keeping the chip at lower peak and average temperatures, integrated on-chip PCMs could potentially improve chip lifetime and reliability.

\section{Acknowledgements}

This work was supported by the National Science Foundation under Grant No. CCF-1161505 and No. CCF-1161681.

\section{References}

[1] A. Abhat, Low-Temperature Latent-Heat Thermal-Energy Storage - Heat-Storage Materials, Sol Energy, 30(4) (1983) 313-332. 
[2] M.M. Farid, A.M. Khudhair, S.A.K. Razack, S. Al-Hallaj, A review on phase change energy storage: materials and applications, Energ Convers Manage, 45(9-10) (2004) 1597-1615.

[3] N. Javani, I. Dincer, G.F. Naterer, B.S. Yilbas, Heat transfer and thermal management with PCMs in a Li-ion battery cell for electric vehicles, Int J Heat Mass Tran, 72 (2014) 690-703.

[4] Z.H. Rao, S.F. Wang, A review of power battery thermal energy management, Renew Sust Energ Rev, 15(9) (2011) 4554-4571.

[5] P. Espeau, D. Mondieig, Y. Haget, M.A. Cuevas-Diarte, 'Active' Package for Thermal Protection of Food Products, Packaging Technology and Science, 10(5) (1997) 253-260.

[6] B. Zalba, J.M. Marin, L.F. Cabeza, H. Mehling, Review on thermal energy storage with phase change: materials, heat transfer analysis and applications, Appl Therm Eng, 23(3) (2003) 251-283.

[7] W.H. Zhao, A.F. Elmozughi, A. Oztekin, S. Neti, Heat transfer analysis of encapsulated phase change material for thermal energy storage, Int J Heat Mass Tran, 63 (2013) 323-335.

[8] V.V. Tyagi, D. Buddhi, PCM thermal storage in buildings: A state of art, Renew Sust Energ Rev, 11(6) (2007) 1146-1166.

[9] R.W. Bonner, T. Desai, F. Gao, X.D. Tang, T. Palacios, S. Shin, M. Kaviany, Die Level Thermal Storage for Improved Cooling of Pulsed Devices, P Ieee Semicond Ther, (2011) 193198.

[10] D. Piedra, T.G. Desai, R. Bonner, M. Sun, T. Palacios, Integration of a Phase Change Material for Junction-Level Cooling in GaN Devices, 2012 28th Annual Ieee Semiconductor Thermal Measurement and Management Symposium (Semi-Therm), (2012) 169-172.

[11] A. Raghavan, Y.X. Luo, A. Chandawalla, M. Papaefthymiou, K.P. Pipe, T.F. Wenisch, M.M.K. Martin, Computational Sprinting, Int S High Perf Comp, (2012) 249-260. 
[12] A. Raghavan, Y.X. Luo, A. Chandawalla, M. Papaefthymiou, K.P. Pipe, T.F. Wenisch, M.M.K. Martin, Designing for Responsiveness with Computational Sprinting, Ieee Micro, 33(3) (2013) 8-15.

[13] A. Raghavan, L. Emurian, L. Shao, M. Papaefthymiou, K.P. Pipe, T.F. Wenisch, M.M.K. Martin, Utilizing Dark Silicon to Save Energy with Computational Sprinting, Ieee Micro, 33(5) (2013) 20-28.

[14] A. Raghavan, L. Emurian, L. Shao, M. Papaefthymiou, K.P. Pipe, T.F. Wenisch, M.M.K. Martin, Computational Sprinting on a Hardware/Software Testbed, Acm Sigplan Notices, 48(4) (2013) 153-166.

[15] C.Y. Zhao, W. Lu, Y. Tian, Heat transfer enhancement for thermal energy storage using metal foams embedded within phase change materials (PCMs), Sol Energy, 84(8) (2010) 14021412.

[16] Z.Q. Chen, D.Y. Gao, J. Shi, Experimental and numerical study on melting of phase change materials in metal foams at pore scale, Int J Heat Mass Tran, 72 (2014) 646-655.

[17] H.X. Ji, D.P. Sellan, M.T. Pettes, X.H. Kong, J.Y. Ji, L. Shi, R.S. Ruoff, Enhanced thermal conductivity of phase change materials with ultrathin-graphite foams for thermal energy storage, Energ Environ Sci, 7(3) (2014) 1185-1192.

[18] A. Mills, M. Farid, J.R. Selman, S. Al-Hallaj, Thermal conductivity enhancement of phase change materials using a graphite matrix, Appl Therm Eng, 26(14-15) (2006) 1652-1661.

[19] O. Sanusi, R. Warzoha, A.S. Fleischer, Energy storage and solidification of paraffin phase change material embedded with graphite nanofibers, Int J Heat Mass Tran, 54(19-20) (2011) 4429-4436. 
[20] S.P. Gurrum, Y.K. Joshi, J. Kim, Thermal management of high temperature pulsed electronics using metallic phase change materials, Numer Heat Tr a-Appl, 42(8) (2002) 777-790. [21] P.J. Shamberger, Cooling Capacity Figure of Merit for Phase Change Materials, Journal of Heat Transfer, 138(2) (2016) 024502.

[22] B. Sarler, Stefan's work on solid-liquid phase changes, Eng Anal Bound Elem, 16(2) (1995) 83-92.

[23] L.N. Tao, The Exact-Solutions of Some Stefan-Problems with Prescribed Heat-Flux, J Appl Mech-T Asme, 48(4) (1981) 732-736.

[24] N.N. Salva, D.A. Tarzia, Explicit solution for a Stefan problem with variable latent heat and constant heat flux boundary conditions, J Math Anal Appl, 379(1) (2011) 240-244.

[25] A. Faghri, Y. Zhang, Transport phenomena in multiphase systems, Elsevier Academic Press, Burlington, Mass., 2006.

[26] See Alloys Sorted by Temperature published by Indium Corporation, Inc.

[27] A. Carroll, H. Gernot. An Analysis of Power Con-sumption in a Smartphone, Proceedings of the 2010 USE-NIX conference on USENIX annual technical conference (2010).

[28] T.E. Bernard, M.F. Foley. Upper Acceptable Surface Temperature for Prolonged Hand Contact, International Journal of Industrial Ergonomics 11(1) (1993) 29-36.

[29] I.C. Ume, J. Gong, Evaluation of Lead-Free Solder Bump Voiding Ball Grid Array Packages Using Laser Ultrasound and Interferometric Technique, Ieee T Comp Pack Man, 3(8) (2013) 1310-1320.

[30] L. Yang, J. Gong, I.C. Ume, Fundamental Study of Microelectronic Chips' Response Under Laser Excitation and Signal Processing Methods, J Nondestruct Eval, 34(3) (2015). 
[31] J. Gong, I.C. Ume, Nondestructive Evaluation of Poor-Wetted Lead-Free Solder Bumps in Ball Grid Array Packages Using Laser Ultrasound and Interferometric Technique, Ieee T Comp Pack Man, 3(8) (2013) 1301-1309.

[32] C. Ume, J. Gong, R. Ahmad, A. Valdes, Laser Ultrasonic Inspection of Solder Bumps in Flip-Chip Packages Using Virtual Chip Package as Reference, Ieee T Comp Pack Man, 1(11) (2011) 1739-1746. 